\title{
Characteristics of the dependences of mobility and concentration of charge carriers in single crystals $\mathrm{CdSb}(\mathrm{In})$ after $\gamma$-irradiation
}

\author{
A.V. Fedosov, Y.V. Koval, L.V. Jashchinskij, O.V. Kovalchuk \\ Lutsk State Technical University, Department of Physics, \\ 75, Lvivska str., 43018 Lutsk, Ukraine; e-mail: jura_koval@gala.net
}

\begin{abstract}
The results of researches of the kinetic effects in crystals $\mathrm{CdSb}$ are presented and discussed.
\end{abstract}

Keywords: crystal CdSb, kinetic effects, electrophysical properties, charge carriers.

Manuscript received 07.02.07; accepted for publication 27.09.07; published online 31.10.07.

The nature of radiation defects and the character of changes of the electrophysical properties of crystals at their irradiation are determined by factors depending on both the properties of irradiated crystals and the doses of irradiation.

In the given work, the kinetic parameters of single crystals of antimonide cadmium alloyed by indium are researched before and after $\gamma$-irradiation. The measurements were conducted on the specimens cut out in basic crystallographic directions. We have studied the specific conductivity and Hall's effect, which allows us to get the dose and temperature dependences of the concentration of charge carriers and their mobility.

The dependence of the concentration of charge carriers on the dose of irradiation is presented in Fig. 1. As can be seen, the changes of the concentration with a dose at room temperature (curve 1, Fig. 1) have a poorly expressed character, because they are the function of the initial concentration of the In admixture $[1,2]$.

As distinct from the case of room temperature, the dependence of the concentration of charge carriers on the dose of irradiation at $78 \mathrm{~K}$ (curve 2, Fig. 1) has a more pronounced character. We refer the concentration decrease, i.e. the removal of electrons from the area of conductivity, to the increase of the concentration of radiation defects with a deep level lying in the forbidden band of $\mathrm{CdSb}(\mathrm{In})$. According to the data of the temperature dependences of the charge carrier concentration in $\gamma$-irradiated crystals (Fig. 2), we assume that the energy level $E_{c}=0.3 \mathrm{eV}$ is responsible for the removal of charge carriers.

The dependences of the charge carrier mobility on the $\gamma$-irradiation dose, $\mu=f(\Phi)$, are presented in Fig. 3 . We see the presence of the maximum at the dose of $4 \cdot 10^{18} \gamma$-quantum $/ \mathrm{cm}^{2}$. We associate the increase of the charge carrier mobility up to a dose of $4 \cdot 10^{18} \gamma$ quantum $/ \mathrm{cm}^{2}$ with the insertion of radiation-induced centers which originate near positively charged admixture (In) ions, where the probability of their appearance is greater because of strains of the lattice near the admixture centers. As a result, the "neutralization" of admixture ions happens, by leading to a decrease of the Rutherford scattering by the partly "neutralized" centers, i.e. to the increase of $\mu$.

Such an explanation can have a place because the probability of the appearance of radiation-induced damages in the strained crystal areas is much higher than that in the relaxed areas [3]. That is why it is natural to expect that, under the low-dose irradiation, the insertion of defects happens mainly in the locally strained areas of the lattice, i.e. near atoms of the dopant (In). This leads finally to the increase of the carrier mobility $\mu$, which is observed in the experiment.

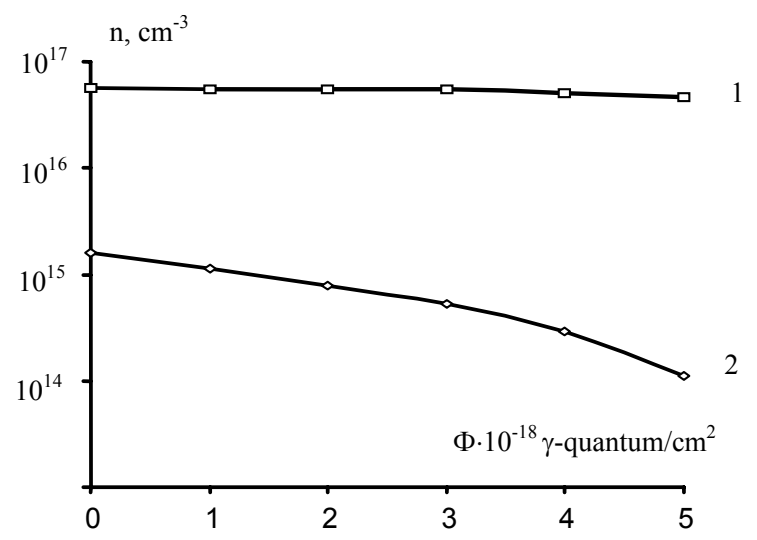

Fig. 1. Dose dependence of the concentration of charge carriers in single crystals $\mathrm{CdSb}(\mathrm{In}): 1$-at $293 \mathrm{~K} ; 2$ - at $78 \mathrm{~K}$. 


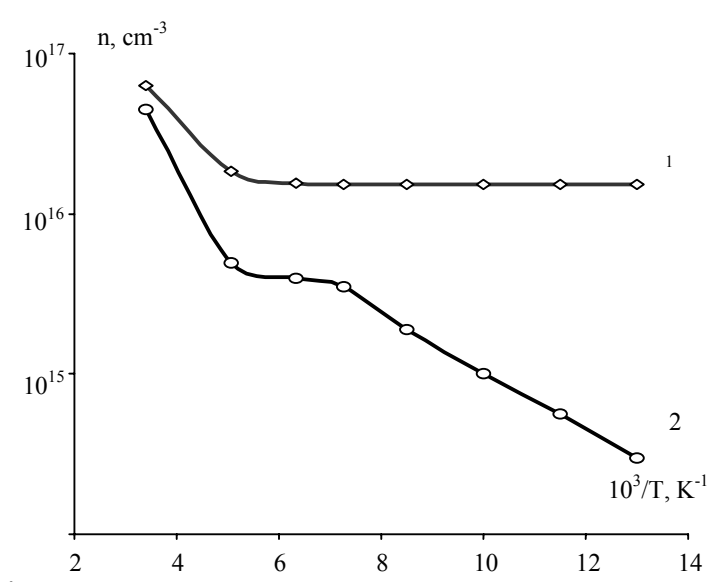

Fig. 2. Iemperature dependence of the charge carrier concentration in single crystals $\mathrm{CdSb}(\mathrm{In}): 1$ - before irradiation; 2 - after an irradiation dose of $4 \cdot 10^{18} \gamma$-quantum $/ \mathrm{cm}^{2}$.

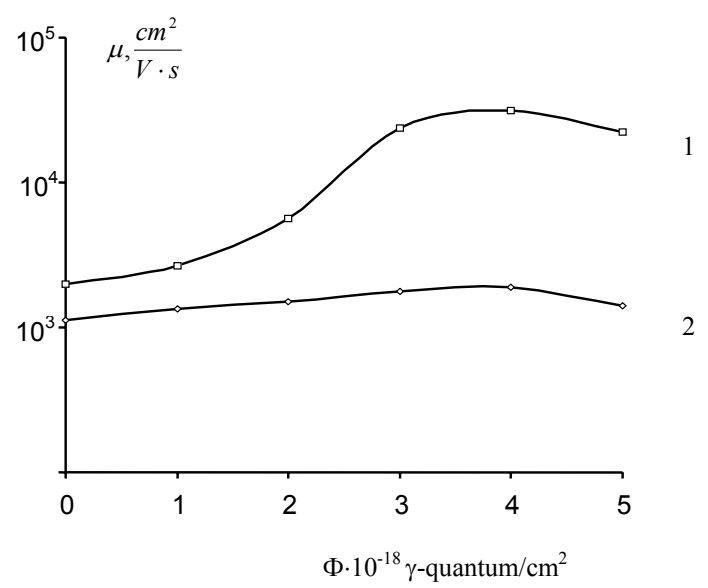

Fig. 3. Dose dependences of the charge carrier mobility in single crystals $\mathrm{CdSb}(\mathrm{In}): 1-$ at $78 \mathrm{~K} ; 2$ - at $293 \mathrm{~K}$.

At the further increase of the irradiation dose, we observe a reduction of $\mu$ after a dose of $4 \cdot 10^{18} \gamma$ quantum $/ \mathrm{cm}^{2}$ at $78 \mathrm{~K}$ (curve 1, Fig. 3), which is conditioned by the scattering by radiation defects.
In addition, it is necessary to note the insignificant increase of $\mu$ with increase in the dose of irradiation at room temperature (curve 2, Fig. 3) as compared with the case of a temperature of $78 \mathrm{~K}$ (curve 1, Fig. 3). Such a behavior of $\mu=f(\Phi)$ is explained by that, at higher temperature, the radiation-induced defects are ionized and practically don't influence the neutralization of admixture ions, which is manifested, in its turn, in the relatively small increase $\mu=f(\Phi)$ in the dose interval up to $4 \cdot 10^{18} \gamma$-quantum $/ \mathrm{cm}^{2}$.

Thus, the radiation centers which arise in the crystal under irradiation both at room and liquid nitrogen temperatures partially "neutralize" the charge of admixture ions which are localized at the lattice points. That is why the charge carrier mobility increase, which is demonstrated by this work, appears not due to the increase of the order of crystals under their irradiation, but due to lowering the effectiveness of the charge carrier scattering by admixture ions on the partial "neutralization" of their charge by radiation-induced defects with the charge of the opposite sign. The obtained results and their interpretation are in agreement with the analogous data presented in the works concerning silicon and germanium single crystals $[3,4]$.

\section{References}

1. E.A. Davis, D.V. Compton, Compensation dependence of impurity conduction in antimonydoped germanium // Phys. Rev. A 140, p. 21832194 (1965).

2. C. Yamanouchi, Effects of the magnetic field on the intermediate impurity conduction in n-Ge // $J$. Phys. Soc. Jpn 18, p. 1775-1784 (1963).

3. E.N. Vidalko, G.P. Gaidar, V.A. Girii, The mobility of current carriers in $\gamma$-irradiated $\mathrm{Si}$ crystals // Izv. AN SSSR, Ser. Neorg. Mat. 22, p. 553-555 (1986) (in Russian).

4. E.N. Vidalko, G.P. Gaidar, V.A. Girii et al., Mobility of majority carriers in $\gamma$-irradiated oxygen-containing crystals of $\mathrm{n}-\mathrm{Si}$ and $\mathrm{n}-\mathrm{Ge} / /$ Phys. status solidi (a) 97, p. 565-570 (1986). 